\title{
COLLABORATIVE ENGINEERING WITH IFC AND BCF: MANAGING OPENINGS AND RECESSES
}

\author{
Léon van Berlo ${ }^{1}$, Mathijs Natrop ${ }^{2}$, and Gerwin Korpershoek ${ }^{3}$
}

\begin{abstract}
In a BIM data flow, an essential part of the coordination between disciplines is the agreement on recesses and openings in building elements. Different disciplines will request recesses and openings in elements that are managed and maintained by others. Using the full dataset of the elements has proven to be inoperable because of the information overload, interoperability problems and contradictions in the commonly used downstream dataflow. A group of companies has been experimenting with the exchange of data that only consists of the requested openings and recesses in IFC. Coordination is being done using BCF. This approach has been fine-tuned and adjusted during several projects. This paper describes the current status of that initiative and discusses the challenges that are still open.
\end{abstract}

Keywords: BIM, coordination, interoperability, exchange.

\section{INTRODUCTION}

In a BIM data flow, an essential part of the coordination between disciplines is the agreement on recesses and openings in elements. Different disciplines will request recesses and openings in elements that are managed and maintained by other disciplines.

The process involves locating equipment and routing connecting elements for each building system. This multidiscipline effort is time-consuming and expensive and requires knowledge regarding each system over the project life cycle (Korman et al. 2003). The coordination of mechanical, electrical, and plumbing (MEP) systems to detail their configuration is a major challenge for complex buildings and industrial projects. Fragmented responsibility for these systems and the knowledge required for their design, installation, and operation make this difficult (Tatum 2000).

\section{TYPICAL PROCESS}

\subsection{From rough design to detailed models}

From the very early moment in a design process, architects like to know what space they need to reserve for construction elements and MEP elements. In the first three phases of a typical design and engineering process (van Berlo et al. 2015), there are many uncertainties to deal with. In this part of the process preferably the Architect, Construction Engineer and MEP advisor are involved. In practise the Construction Engineer and the MEP engineer only get involved during the creation of a model on Information level 2 or 3.

1 Netherlands organisation for applied scientific research TNO, Delft, The Netherlands, leon.vanberlo@tno.nl

$2 \quad$ BuildingSMART Benelux chapter, The Netherlands, mathijs.natrop@buildingsmart.nl

$3 \quad$ BIM Manager, Unica, The Netherlands, gkorpershoek@unica.nl 
A construction engineer indicates overall sizes for beams and columns and the thickness of the floors. The MEP advisor indicates the size of the technical spaces, sizes of the shafts and piping, and the necessary space between the ceiling and the floor. In this phase, only global mass geometries are being modelled and exchanged. The focus is on mass study and visualisation. The result is a model on Information Level 1 which is the base for a design on Information Level 2 (van Berlo et al. 2015) which is typically called a 'Preliminary Design'. In this phase the principle design decisions are made regarding the construction design and MEP design. Individual elements will be modelled, but only on a principle level meaning they will not have the actual size, material, etc. This will give a first indication of the individual piping and individual building elements. At this moment in the design process, the intersections between objects from different disciplines can be evaluated. Critical points of the intersections, mainly between construction elements and MEP elements, get extra attention from the design team in this phase. Based on experience and practical knowledge, the critical points are modelled in more detail and discussed during the coordination sessions.

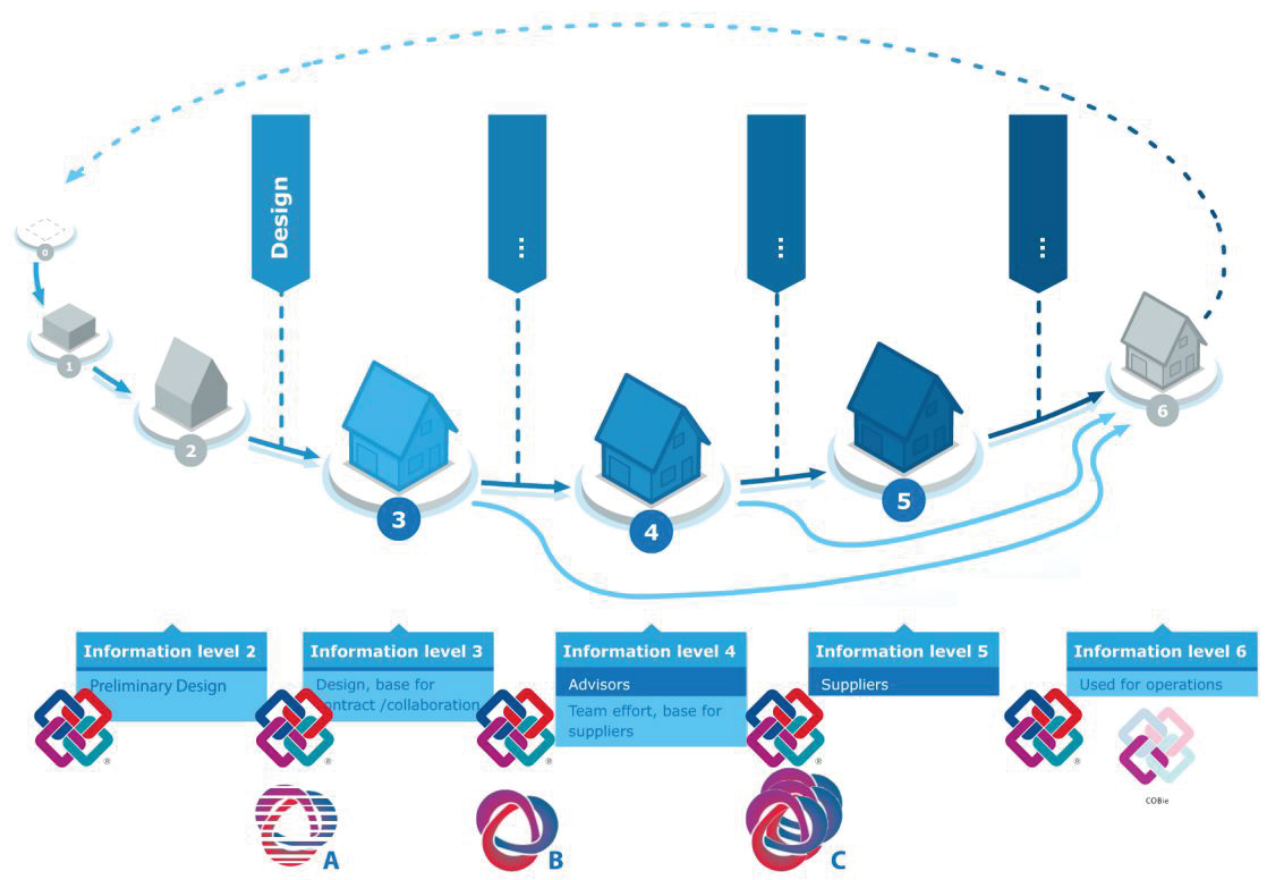

Figure 1: phases of design and engineering and use of IFC and BCF for coordination of openings and recesses.

In the next phase (in figure 1 indicated as 'A') all the objects indicating space reservations will be replaced with actual beams, columns, floors, air ducts, piping, trays, equipment, etc. In complex projects this is the first-time openings and recesses between disciplines are being generated and exchanged in a separate IFC file. An example of such a dataset is shown in figure 3. In some advanced projects, we observed the use of BCF in this phase as well, but not specific for the coordination of openings and recesses.

After some design iterations, a model on Information Level 3, typically called a 'Definitive Design', is finalized.

The next phase involves several other advisors and engineers to get the model to a more detailed level. This phase is usually led by the contractor. The model resulting from this phase is the base for suppliers to specify their solutions. In this phase (in Figure 1 
indicated with a 'B') intense coordination on a detailed level is performed between engineers from several disciplines. More information is needed about the openings and recesses than in the previous phases. Use of BCF specifically for coordination about openings and recesses is very common.

After this phase the final engineering phase (in Figure 1 indicated with a ' $\mathrm{C}$ ') is starting: suppliers deliver their aspect models with the information on what they are going to produce and deliver. In this phase the final openings and recesses from objects and elements are being engineered to a final result. Coordination about the status of the openings and elements is vital in this phase and is being done using BCF.

\subsection{Issues}

In a typical process described above, there is a paradox in the information needed and the information available. During the coordination of the design phase (preliminary design and final design) the main focus is on geometry. There is a need for information about the size of openings to further detail the elements, but other disciplines need more information about the elements before they can provide information about the necessary recesses and openings. This 'catch 22'-situation can only be breached with intense coordination and short design iterations.

During the engineering phase (called 'B' in Figure 1), many different companies are involved in the engineering. The coordination between all the organisations is intense and a reliable process for managing the openings and recesses is vital for the progression of this phase. In this phase, not only the geometry of the openings and recesses is important, but also other information (what is the reason for the opening? Does it need to be airtight? Does it need to be fire proof?) is needed. Interoperability between many different software tools is a key issue during this phase.

In the phase where suppliers are involved (called ' $\mathrm{C}$ ' in figure 1), the information about recesses and openings is being finalized. During the many iterations in this phase the status of the openings and recesses is crucial to participants.

\section{INTEROPERABILITY}

Van Berlo et al. showed the use of IFC in a downstream workflow (2012) as very effective. Later (2015) is was showed to be the 'de facto' standard of BIM collaboration in the Netherlands. Base principle of this collaboration workflow is the exchange of data that are needed for others to do their work. In the case about openings and recesses it makes perfect sense to only exchange opening and recesses to team members.

The fragmentation of disciplines that all have requests for openings and recesses is typically very high. The paradox situation during the design phase needs to be breached as soon as possible. This agues for an exchange of information about openings and recesses as early as possible and as often as possible. The agile necessity of coordinating openings and recesses is demanding a flexible approach of information exchange. These are the main reasons why the industry is using separate aspect models to exchange just openings and recesses. This is almost always done in IFC. An example of such a dataset is shown in Figure 3.

Some tools in the MEP sector have specific functions to generate the openings and recesses for ducts, piping, etc. A geometric object is created for the requested opening, including additional information about the status, type of opening, etc. An example of this is shown in Figure 2. However, not all tools have these functions and the exchange 
of this information is a proprietary XML file. The industry is seeking the advantages of exchange with an open standard like BCF.

\section{OBSERVATIONS AND EXAMPLES}

This chapter shows some examples of the workflow for creation, exchange and coordination of openings and recesses. Observations and issues are given in each example.

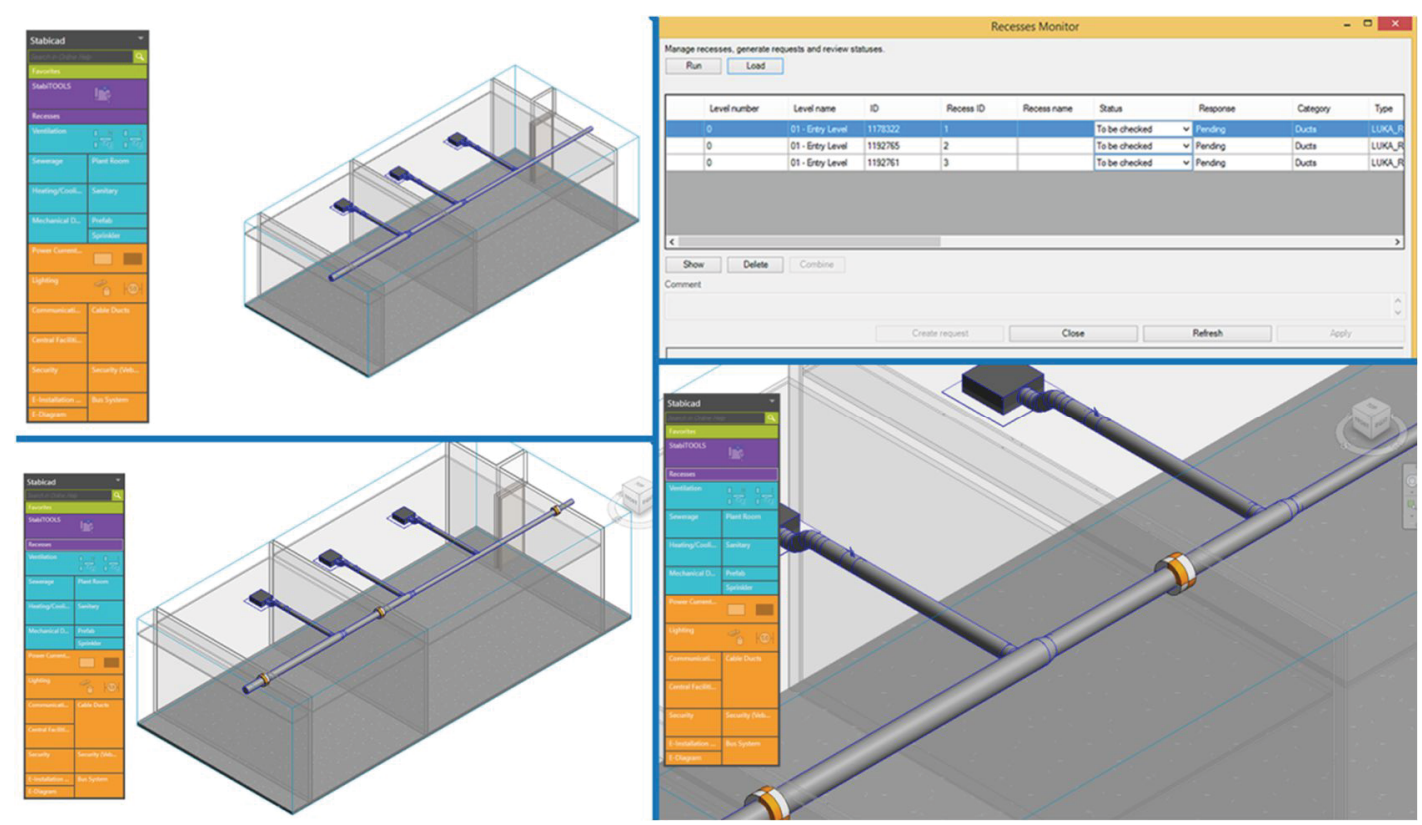

Figure 2a: Example of the generation of a 'recesses request' in Revit/Stabicad

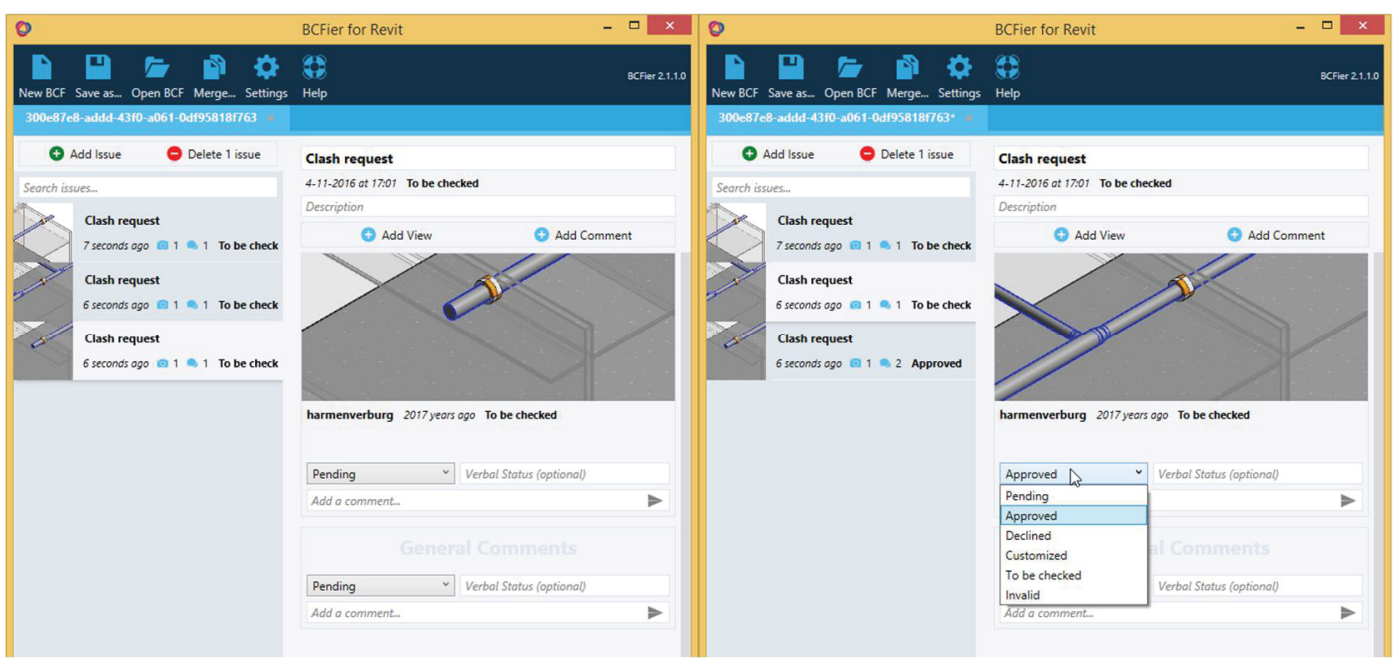

Figure 2b: example of the $\mathrm{BCF}$ topic regarding the requests and the alteration of the status 

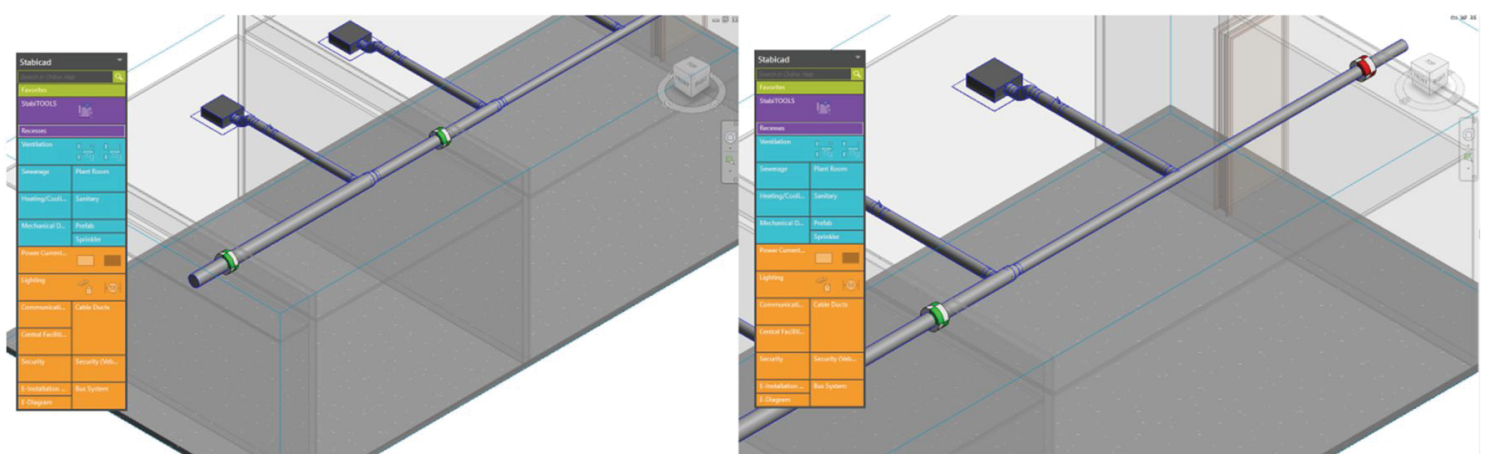

Figure 2c: Result of the altering of the status visualized in Revit/Stabicad

Figure 2a is showing the process from the perspective of the MEP designer/engineer. In Revit (Stabicad) the engineer creates a 'request' for recesses in the walls. Stabicad generates the geometry of the requested openings. This information can be exported as IFC and BCF.

Adaptation of the status is shown in Figure 2b. This is shown using BCFier, an open source $\mathrm{BCF}$ tool with a plugin for Revit. In this example, another engineer (could be the coordinator, architect or structural engineer) is evaluating the requests by changing the status of the BCF topic. There is no need to use Revit or Stabicad for this coordination.

After the status, has changed the updated $\mathrm{BCF}$ is returned to the MEP engineer who imports it in Revit/Stabicad. Figure 2c shows the result of the import where two of the requests have been approved and one is rejected.

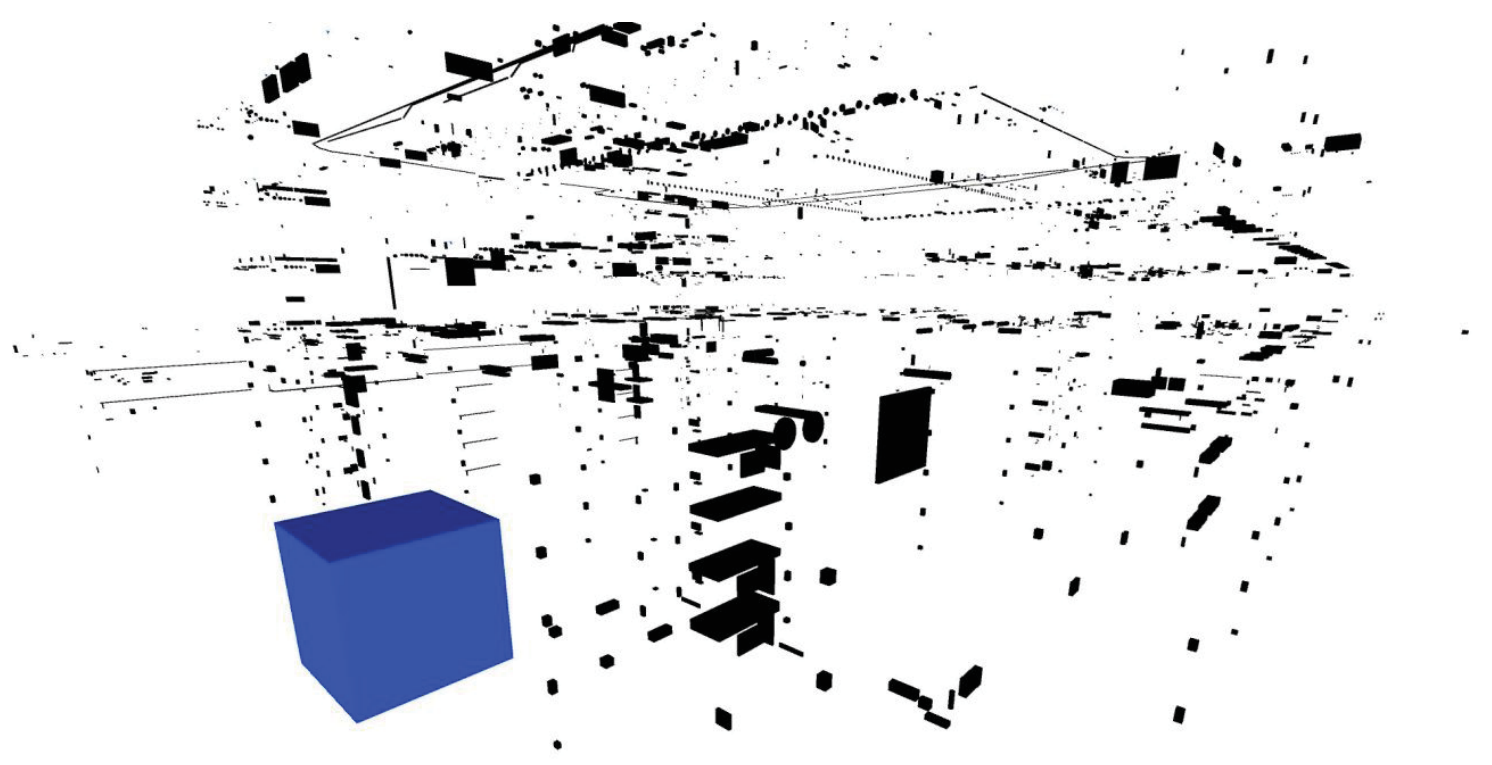

Figure 3: visualisation of an IFC dataset with only

(requested/approved/designed/etc.) openings and recesses. The big blue box is the shared origin point used to align the different aspect models. 


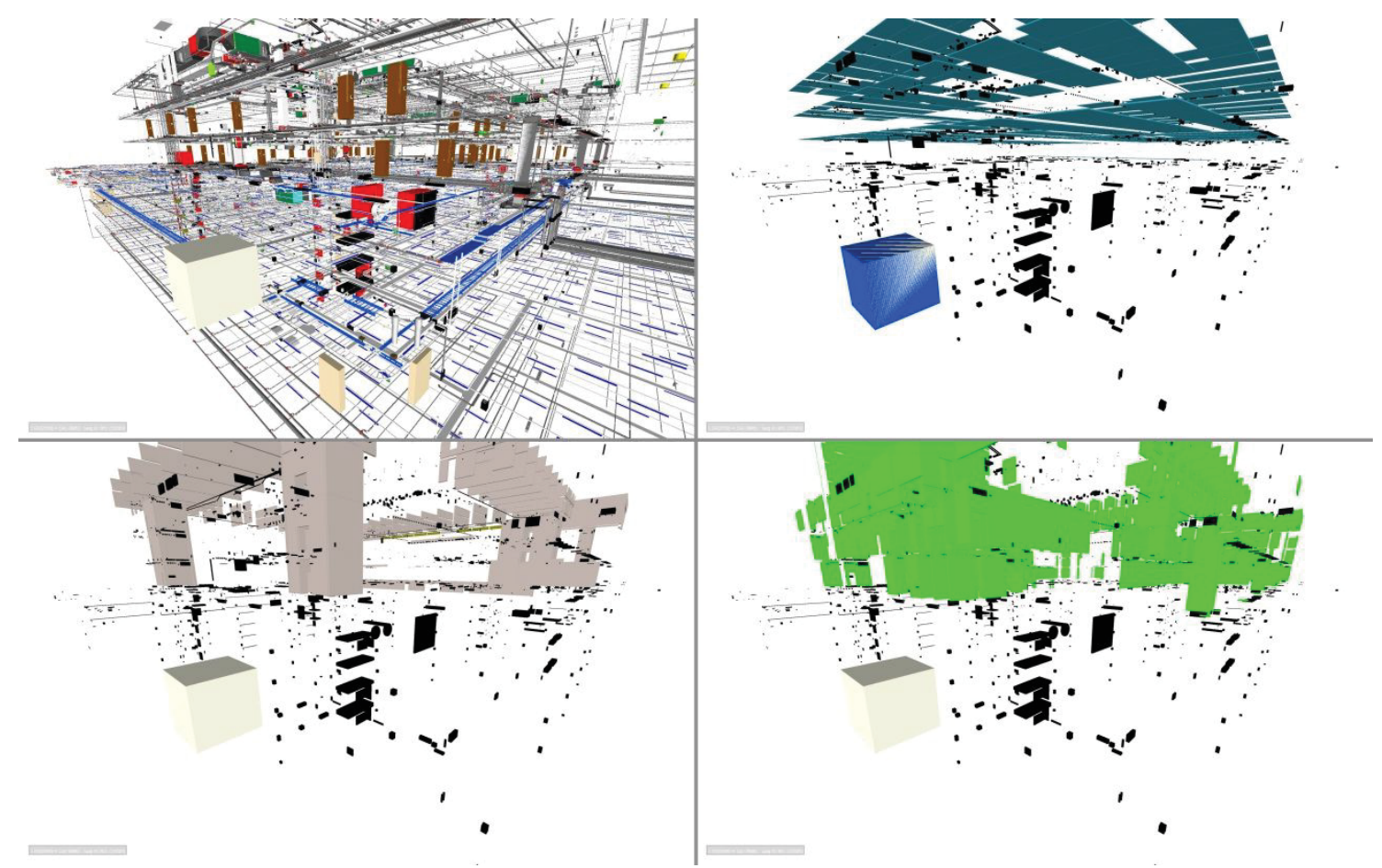

Figure 4: The same openings and recesses as in Figure 2, but now with (starting upper left, going clockwise) the full MEP aspect model, the aspect model of the floors, the aspect model of the limestone, the aspect model of the in-situ concrete. In these pictures the big box is the shared origin point that is used for alignment of the aspect models.

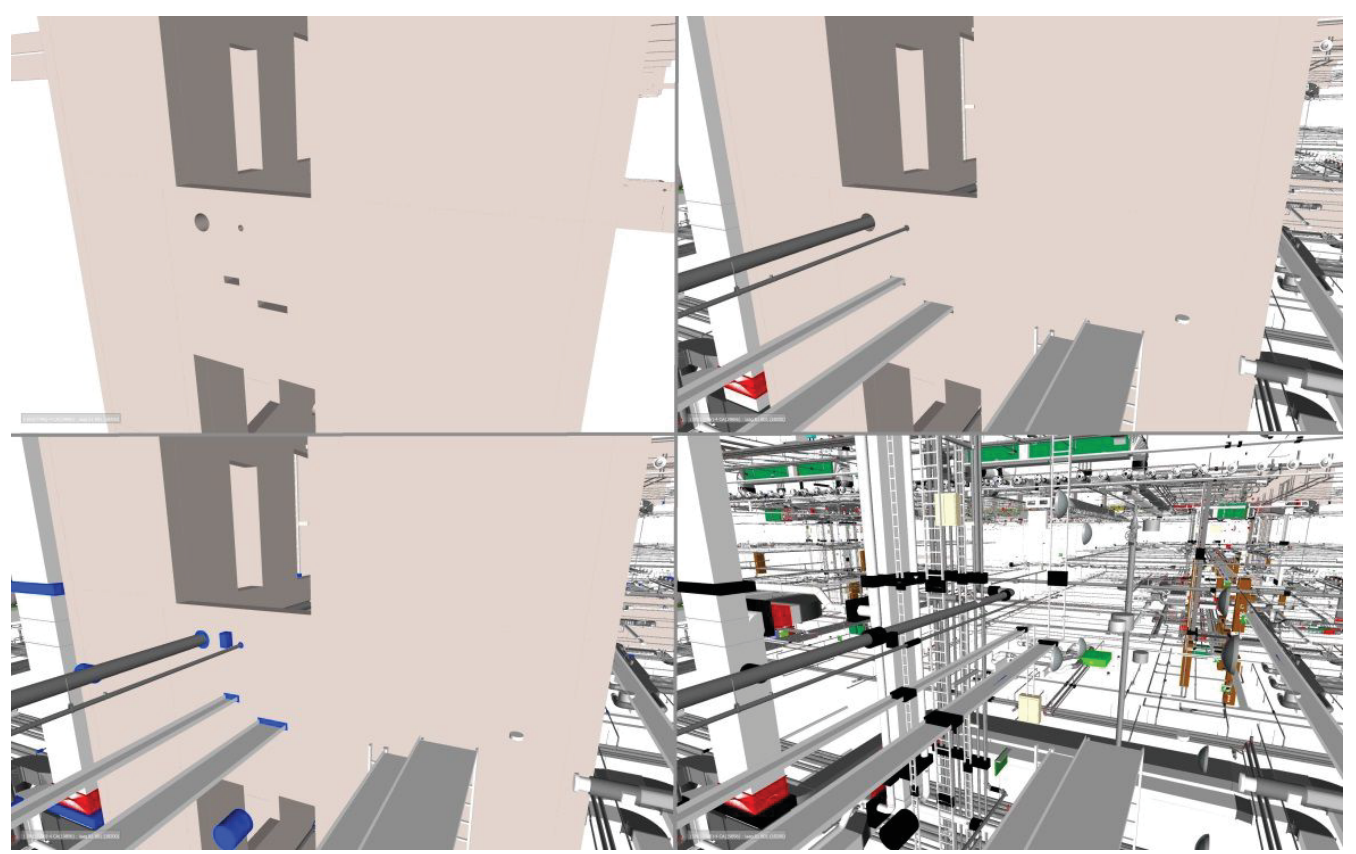

Figure 5: showing the same situation with (starting upper left, going clockwise) only the walls, walls and MEP, only MEP, walls and MEP and openings/ recesses aspect models. 
Figures 3 to 6 are from the same project. This project has over 250 aspect models in the engineering phase. According to the BIM coordinator it would be impossible to coordinate openings and recesses between the different disciplines by exchanging the full datasets. Using a specific dataset for only openings and recesses created the ability to coordinate more often and earlier in the process. Thereby reaching a faster consensus about the openings and recesses which makes it able to finalize the engineering faster and avoiding the issues described by Sacks et al. (2011).

\section{Challenges}

During the observations and applied research at real projects, the authors found some challenges that still need to be addressed.

1. Openings or recesses that have been moved from location (or are proposed on a different location) are difficult to coordinate. This is mainly due to the issue that GUIDs of BCF topics change and it is impossible for a computer to match new topics against older topics when they don't have the same identifier and location.

2. There is no common process for merging openings/recesses. When several disciplines use the same opening for multiple ducts or piping, the coordination of the single opening element is facing practical challenges.

3. Due to the file based exchange of BCF topics it is impossible to merge multiple comments on the same topics that are being generated by multiple disciplines. Berlo \& Krijnen (2014) and Treldal et.al. (2016) already found this limitation and proposed solutions in the architecture of the BCF standard for this.

The authors agree that the status of the described workflow is not final yet. Collaboration between researchers and practice will need to continue to create a final consensus about this approach.

\section{RECOMMENDATIONS}

The authors see a need to continue the discussion and testing the use of $\mathrm{BCF}$ for this coordination. Suggestions are:

- Standardising the enumeration for exchanging status about openings and recesses. This enumeration should not conflict with commonly used status enumerations of building elements coordination.

- Testing the use of IFC snippets with BCF to experiment transitions of BCF topics (described in challenges 1 and 2).

The authors like to note that many of the interviews people stressed their recommendation to avoid a discussion about Model View Definitions and IFC Extensions.

\section{CONCLUSIONS}

This research concludes that the separate exchange of aspect models for opening and recesses with IFC is an effective approach for BIM coordination (both during design, and engineering).

The authors describe a process where a team works from rough geometries towards more detailed geometry, towards geometry with attributes, to sharing status information 
about objects. Interviews have indicated that this is the preferred and most effective workflow for a BIM project.

Although some challenges are still open, the use of BCF to exchange status information about IFC objects has proved to be the most promising technology for coordination of openings and recesses. This conclusion is with the proviso that the new versions of the BCF standard find solutions for the stated challenges.

On general this research confirms that the implementation of BIM based tools in construction organizations is a "technology pull" (Hartmann et al, 2012).

\section{DISCUSSION}

This research has used the method of interviews, observations and applied research in practice. The number of projects that this publication was based upon was limited. More quantitative and qualitative research needs to be performed to validate the conclusions in this paper.

\section{REFERENCES}

Hartmann, T., Van Meerveld, H., Vossebeld, N. and Adriaanse, A. (2012). Aligning building information model tools and construction management methods. Automation in construction, 22, 605-613.

Korman, T. M., Fischer, M. A. and Tatum, C. B. (2003). Knowledge and reasoning for MEP coordination. Journal of Construction Engineering and Management, 129(6), 627-634.

Sacks, R. and Melhado, S. B. (2011). Key performance indicators to analyze and improve management of information flow in the BIM design process. Proceedings of the CIB W78-W102.

Tatum, C. B. and Korman, T. (2000). Coordinating building systems: process and knowledge. Journal of Architectural Engineering, 6(4), 116-121.

Treldal, N., Parsianfar, H. and Karlshøj, J. (2016) Using BCF as a mediator for task management in building design.

van Berlo, L. A. H. M., Beetz, J., Bos, P., Hendriks, H. and Van Tongeren, R. C. J. (2012). Collaborative engineering with IFC: new insights and technology. In 9th European Conference on Product and Process Modelling, Iceland.

van Berlo, L. and Krijnen, T. (2014). Using the BIM collaboration format in a server based workflow. Procedia Environmental Sciences, 22, 325-332.

van Berlo, L., Derks, G., Pennavaire, C. and Bos, P. (2015) Collaborative Engineering with IFC: common practice in the Netherlands. Proc. of the 32nd CIB W78 Conference 2015, October 27th-29th 2015, Eindhoven, The Netherlands. 